\title{
Steffen Hendel, Den Krieg erzählen
}

\section{Chloé Chaudet}

\section{OpenEdition \\ Journals}

Édition électronique

URL : http://journals.openedition.org/ifha/9324

DOl : $10.4000 /$ ifha. 9324

ISSN : 2198-8943

Éditeur

IFRA - Institut franco-allemand (sciences historiques et sociales)

\section{Référence électronique}

Chloé Chaudet, «Steffen Hendel, Den Krieg erzählen », Revue de l'IFHA [En ligne], Date de recension, mis en ligne le 27 juillet 2018, consulté le 24 septembre 2020. URL : http://journals.openedition.org/ ifha/9324 ; DOl : https://doi.org/10.4000/ifha.9324

Ce document a été généré automatiquement le 24 septembre 2020.

(C)IFHA 


\section{Steffen Hendel, Den Krieg erzählen}

\section{Chloé Chaudet}

\section{RÉFÉRENCE}

Steffen Hendel, Den Krieg erzählen. Positionen und Poetiken der Darstellung des Jugoslawienkrieges in der deutschen Literatur, Osnabrück: V\&R unipress, 2017, 348 p., $50,00 €$ 
Dans cet ouvrage issu de sa thèse de germanistique, Steffen Hendel questionne les positions de la « littérature allemande réunifiée » (p.17) face à un changement fondamental qui s'est produit dans les années 1990: l'irruption de la violence militaire comme option politique dans les débats intellectuels et les actions politiques allemandes. Prenant pour objet d'étude principal les textes littéraires créés à partir de cette période, qui succèdent à une littérature d'après-guerre (« Nachkriegsliteratur») se caractérisant par sa critique constitutive de la Seconde Guerre mondiale, S. Hendel analyse les reconfigurations $\mathrm{du}$ champ littéraire germanophone face aux «nouvelles guerres» (p.19) dans lesquelles l'Allemagne a été impliquée au tournant

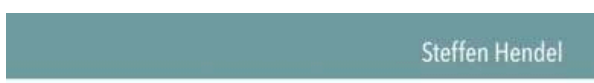
Steffen Hendel du XXI ${ }^{\mathrm{e}}$ siècle.

Le premier contexte abordé est celui de la Deuxième Guerre du Golfe (1990-1991), qui cristallise des débats préliminaires autour de la légitimité de l'interventionnisme militaire au moment où l'Allemagne, tout juste réunifiée, se cherche une identité politique. À partir du deuxième chapitre, S. Hendel se focalise sur le contexte le plus largement traité au sein de son étude : l'éclatement de la Yougoslavie, à partir de l'indépendance de la Slovénie et de la Croatie (1991-1992) jusqu'à celle du Kosovo en 1999, en passant par la guerre de Bosnie-Herzégovine (1992-1995). Enfin, dans un cinquième et dernier chapitre questionnant "l'inventaire des résultats de la guerre » (p. 299), S. Hendel se concentre sur la littérature créée après les guerres de Yougoslavie.

Parallèlement aux mutations de l'opinion publique auxquelles il fait partiellement écho, le champ littéraire allemand se caractérise par une révision ponctuelle du consensus pacifiste d'après-guerre dès le début des années 1990. S. Hendel le montre en prenant pour exemple le recueil Europa im Krieg édité par Willi Winkler en 1992, qui intègre des textes de Herta Müller, Hans Magnus Enzensberger, Lothar Baier ou encore Dunja Melcic. On mentionnera par exemple le texte "Die Tage werden weitergehen » de Herta Müller, où celle-ci, selon une veine polémique, souligne l'absurdité de l'opposition de la plupart des dirigeants d'Europe occidentale à une intervention militaire en Croatie.

S. Hendel associe ensuite la guerre de Bosnie-Herzégovine à une période de "prise de position partisane» (p.92) de l'opinion publique allemande, et analyse à ce titre l'œuvre d'écrivains tels que Peter Schneider, Otmar Jenner ou encore Walter Wüllenwerber, dont l'écriture fait in fine émerger « la légitimité illimitée de l'Allemagne [réunifiée] de déclencher une guerre en cas de nécessité absolue » (p. 125). Mais il se focalise également sur Peter Handke, dont le projet politique et poétique dans le Voyage hivernal, récit des impressions qu'il a recueillies dans la Serbie de 1995, se construit à rebours de discours véhiculés par les médias ouest-européens, qualifiés par l'écrivain autrichien de « bouffeur[s] de Serbes ». En traitant ces diverses positions dans un même 
chapitre, S. Hendel s'inscrit dans une démarche de différenciation des représentations, qu'il considère comme trop négligée par les orientations narratologiques inspirées des études culturelles au sein de la germanistique des dix dernières années (p. 28-29).

La suite de l'ouvrage se concentre sur la période succédant à la décision de l'État allemand de participer à l'intervention de l'OTAN au Kosovo, globalement soutenue par l'opinion publique allemande. Dans ce contexte, S. Hendel met au jour ce qu'il qualifie de «nouvelle qualité » des débats (p. 212) : les éléments de problématisation de leur propre discours par les écrivains. On pourrait objecter que cette dynamique générale caractérisait déjà le Voyage hivernal handkéen, mais l'analyse de textes de Sibylle Berg, de Franz Schirrmacher, de Christian Kracht ou encore de Thomas Meinecke montre de manière précise que l'écriture de la guerre de la fin des années 1990 peut servir de prétexte à une mise en question de la subjectivation du regard de l'écrivain, à l'esquisse d'une éthique valable pour notre ère postmoderne ou à une critique de la langue.

La dernière partie de l'ouvrage est consacrée à la plus récente période de «justification des guerres passées par leurs résultats » (p. 297). Les guerres yougoslaves disparaissent du débat public allemand à la suite de la reconnaissance de l'indépendance du Kosovo en 1999, qui marque la fin de l'éclatement de la Yougoslavie; mais elles continuent à intéresser des écrivain.e.s tels que Terezia Mora, Norbert Gstrein, Sasa Stanisic ou encore Julie Zeh, qui narre son voyage en Bosnie dans un texte dont il n'existe pas encore de traduction française, Die Stille ist ein Geräusch (2002). Leurs œuvres ont pour point commun de porter un bilan négatif sur les conflits passés, en mettant en lumière des figures traumatisées, incapables de s'intégrer dans une communauté civile pacifiée - traumatisme qui concerne même, dans une moindre mesure, des personnages indirectement confrontés à la guerre (p. 299). L'absence d'injonction politico-morale que discerne néanmoins $\mathrm{S}$. Hendel dans ces œuvres écrites après les conflits yougoslaves le mène à interpréter l'historicisation à laquelle elles procèdent selon la formule suivante, sur laquelle se conclut son étude: "Qui veut la paix ne doit pas l'exposer en protestant contre la guerre, mais en se portant responsable de celle-ci et de ce qu'elle produit » (p. 327).

Par son étude sociocritique inédite, S. Hendel livre un tableau représentatif des textes et auteur.e.s concernant et concernés par la période des guerres yougoslaves. Outre les incontournables Peter Handke et Julie Zeh, il convoque des auteur.e.s et/ou textes un peu moins connus à l'échelle européenne pour construire un dialogue fructueux, orienté par une ambition surplombante qui ne nie pas pour autant leurs frictions politiques et poétiques. L'analyse de ce corpus conséquent, et varié au plan générique, fait ainsi émerger de manière nuancée l'évolution des positions des écrivain.e.s germanophones face à un processus de transformation de l'opinion publique allemande, qui mène à ce que la guerre ne soit plus considérée comme un tabou, mais comme une possibilité voire une nécessité politique. 
INDEX

Index chronologique : Époque contemporaine

Thèmes : Histoire des mentalités, histoire de la culture

\section{AUTEURS}

\section{CHLOÉ CHAUDET}

Université Clermont-Auvergne

CELIS 